Fischer, Sabine; Baey-Ernsten, Max de

\title{
Didaktische Perspektiven einer ästhetischen Werkstatt
}

Holub, Barbara [Hrsg.]; Himpsl-Gutermann, Klaus [Hrsg.]; Mittlböck, Katharina [Hrsg.]; Musilek-Hofer, Monika [Hrsg.]; Varelija-Gerber, Andrea [Hrsg.]; Grünberger, Nina [Hrsg.]: lern.medien.werk.statt. Hochschullernwerkstätten in der Digitalität. Bad Heilbrunn : Verlag Julius Klinkhardt 2021, S. 273-290. - (Lernen und Studieren in Lernwerkstätten)

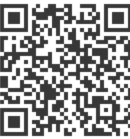

\section{Quellenangabe/ Reference:}

Fischer, Sabine; Baey-Ernsten, Max de: Didaktische Perspektiven einer ästhetischen Werkstatt - In: Holub, Barbara [Hrsg.]; Himpsl-Gutermann, Klaus [Hrsg.]; Mittlböck, Katharina [Hrsg.]; Musilek-Hofer, Monika [Hrsg.]; Varelija-Gerber, Andrea [Hrsg.]; Grünberger, Nina [Hrsg.]: lern.medien.werk.statt. Hochschullernwerkstätten in der Digitalität. Bad Heilbrunn : Verlag Julius Klinkhardt 2021, S. 273-290 - URN: urn:nbn:de:0111-pedocs-228253 - DOI: 10.25656/01:22825

https://nbn-resolving.org/urn:nbn:de:0111-pedocs-228253

https://doi.org/10.25656/01:22825

in Kooperation mit / in cooperation with:

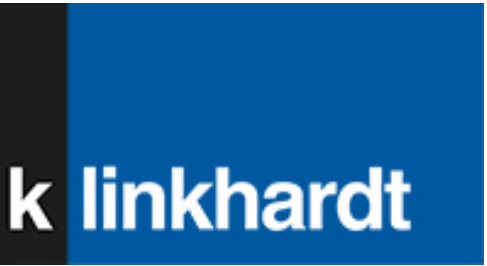

http://www.klinkhardt.de

\section{Nutzungsbedingungen}

Dieses Dokument steht unter folgender Creative Commons-Lizenz: http://creativecommons.org/licenses/by-nc-sa/4.0/deed.de - Sie dürfen das Werk bzw. den Inhalt unter folgenden Bedingungen vervielfältigen, verbreiten und öffentlich zugänglich machen sowie Abwandlungen und Bearbeitungen des Werkes bzw. Inhaltes anfertigen: Sie müssen den Namen des Autors/Rechteinhabers in der von ihm festgelegten Weise nennen. Dies Werk bzw. der Inhalt darf nicht für kommerzielle Zwecke verwendet werden. Die neu entstandenen Werke bzw. Inhalte dürfen nur unter Verwendung von izenzbedingungen weitergegeben werden, die mit denen dieses Lizenzvertrages identisch oder vergleichbar sind.

Mit der Verwendung dieses Dokuments erkennen Sie die Nutzungsbedingungen an.

\section{Terms of use}

This document is published under following Creative Commons-License: http://creativecommons.org/licenses/by-nc-sa/4.0/deed.en - You may copy, distribute and transmit, adapt or exhibit the work in the public and alter, transform or change this work as long as you attribute the work in the manner specified by the author or licensor. You are not allowed to make commercial use of the work. If you alter, transform, or change this work in any way, you may distribute the resulting work only under this or a comparable license.

By using this particular document, you accept the above-stated conditions of

\section{Kontakt / Contact:}

\section{peDOCS}

DIPF | Leibniz-Institut für Bildungsforschung und Bildungsinformation Informationszentrum (IZ) Bildung

E-Mail: pedocs@dipf.de

Internet: www.pedocs.de

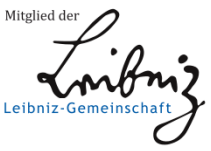




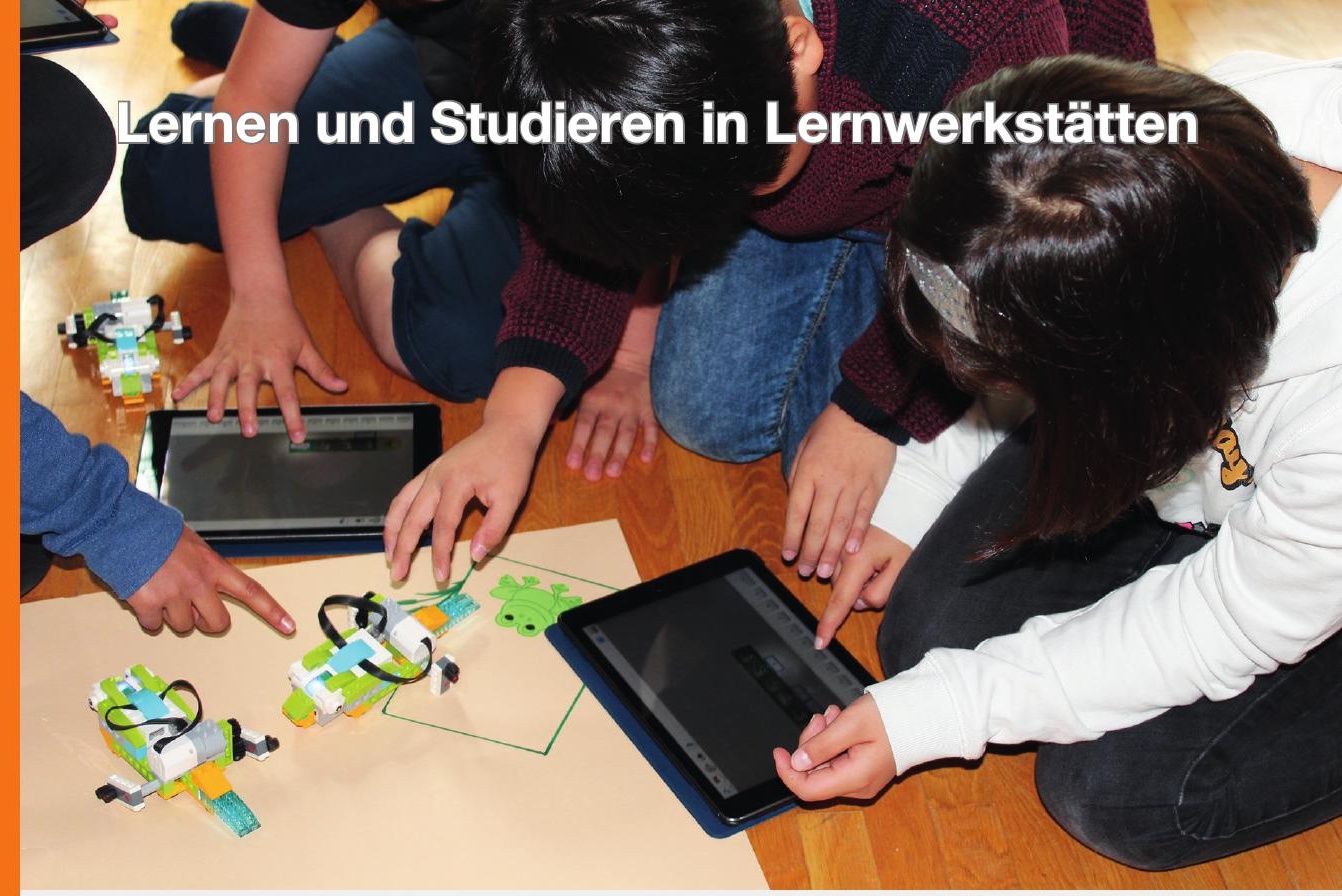

Barbara Holub / Klaus Himpsl-Gutermann Katharina Mittlböck / Monika Musilek-Hofer Andrea Varelija-Gerber / Nina Grünberger (Hrsg.)

\section{lern.medien.werk.statt}

Hochschullernwerkstätten in der Digitalität 
Barbara Holub Klaus Himpsl-Gutermann Katharina Mittlböck Monika Musilek-Hofer Andrea Varelija-Gerber

Nina Grünberger (Hrsg.)

\section{lern.medien.werk.statt}

Hochschullernwerkstätten in der Digitalität 
Dieser Titel wurde in das Programm des Verlages mittels eines Peer-Review-Verfahrens aufgenommen. Für weitere Informationen siehe www.klinkhardt.de.

Bibliografische Information der Deutschen Nationalbibliothek Die Deutsche Nationalbibliothek verzeichnet diese Publikation in der Deutschen Nationalbibliografie; detaillierte bibliografische Daten sind im Internet abrufbar über http://dnb.d-nb.de.

2021.ig. (C) by Julius Klinkhardt.

Coverfoto: (c) ZLI PH Wien.

Druck und Bindung: AZ Druck und Datentechnik, Kempten.

Printed in Germany 2021.

Gedruckt auf chlorfrei gebleichtem alterungsbeständigem Papier.

Die Publikation (mit Ausnahme aller Fotos, Grafiken und Abbildungen) ist veröffentlicht unter der Creative Commons-Lizenz: CC BY-NC-SA 4.0 International https://creativecommons.org/licenses/by-nc-sa/4.0/

ISBN 978-3-7815-5904-2 digital doi.org/10.35468/5904

ISBN 978-3-7815-2468-2 print 


\section{Inhaltsverzeichnis}

Barbara Holub, Klaus Himpsl-Gutermann, Katharina Mittlböck, Monika Musilek-Hofer, Andrea Varelija-Gerber und Nina Grünberger

Einführung in den Band 11

\section{Zur Konstituierung von Hochschullernwerkstätten}

Sandra Tänzer

Mut - Zumutung - Ermutigung. Oder wie man lernt, eine Hochschullernwerkstatt zu lieben

Markus Peschel, Hartmut Wedekind, Pascal Kihm und Mareike Kelkel

Hochschullernwerkstätten und Lernwerkstätten -

Verortung in didaktischen Diskursen

Dietlinde Rumpf und Corinna Schmude

Von der Herausforderung, die Vielfalt von Hochschullernwerkstätten

in einer Definition abzubilden

Pascal Kihm und Markus Peschel

„Komplexität wagen!“ - Methoden zur Beforschung von

offenen Lehr-Lern-Prozessen in Hochschullernwerkstätten 70

\section{Zum Spannungsverhältnis von Hochschullernwerkstätten und Digitalität}

Mark Weißhaupt, Ralf Schneider, Clemens Griesel und Agnes Pfrang

Digitale Erfahrung? Über das Lernen zwischen Instruktion und

(Ko-)Konstruktion

Stefan Brée, Lena S. Kaiser und Tanja Wittenberg

Lernwerkstatt als digitaler Erfahrungsort. Potenziale und

Herausforderungen für Lernwerkstätten als Orte

„offener multimedialer Produktionsästhetik“

\section{Ulrike Stadler-Altmann und Gerda Winkler}

Real \& virtuell, analog \& digital: Dimensionen einer Kooperation.

Multifunktionalität als Kennzeichen zukunftsfähiger Lernwerkstatt- und

Bibliothekskonzeption 


\section{Josef Buchner und Michael Kerres}

Lernwerkstattarbeit in der digital vernetzten Welt.

Die Perspektive der gestaltungsorientierten Mediendidaktik

Sabrina Tietjen und Silvia Thünemann

Forschungswerkstatt digital: ein modernes Lehr-Lernarrangement

für eine Digitalisierungsstrategie im Lehramt?

\section{Zu fach- und mediendidaktischen Perspektiven}

Johannes Mayer, Antonia Lemensieck, Maria Reinhardt und Karl Wollmann

Fachliche Perspektiven auf digitalisierungsbezogene Lernangebote

in der Ausbildung von Grundschullehrer*innen

Heike Hagelgans

Die Thematisierung digitaler Medien in den schulpraktischen Studien

Fachdidaktische Reflexionen von digitalen Medien für das Lernen im

Mathematikunterricht der Primarstufe

Jeanette Hoffmann, Katharina Egerer und Franziska Herrmann

Analoge Bilder - digitaler Film.

Möglichkeitsräume für literarästhetisches Lernen in

Hochschullernwerkstätten

Michael Rieseneder und Wolfgang Wagner

Erstes Programmieren mit Kindern über Handlungserfahrungen.

Das Konzept Activity-based-Coding

Patrick Isele und Julia Höke

Reflexionen über digitales Lernen mit dem Sphero SPRK+

Erfahrungen mit Studierenden in der „Lernwerkstatt ${ }^{3 “}$

Susanne Schumacher, Ulrike Stadler-Altmann und Enrico A. Emili

Piktogramme als Unterstützungsmedien.

Studien zu Effekten von Bilderbüchern mit zusätzlichen Symbolen im Kindergarten 


\section{Zu Aspekten, Perspektiven und Herausforderungen von Hochschullernwerkstätten}

Sabine Fischer und Max de Baey-Ernsten

Didaktische Perspektiven einer ästhetischen Werkstatt

Tanja Wittenberg und Lena S. Kaiser

„Ich war frustriert, ich bin kein Kind mehr“ - Erfahrungslernprozesse

mit verwendungs- und bedeutungsoffenem Material in

kindheitspädagogischen Hochschullernwerkstätten

Lisa Eßel und Laura Schlichting

Hochschullernwerkstätten im Spannungsfeld zwischen

Freiwilligkeit und Pflicht

Mareike Kelkel, Markus Peschel und Pascal Kihm

Potenziale der pädagogisch-didaktischen Öffnung in

Hochschullernwerkstätten

Barbara Holub und Sybille Roszner

Hochschullernwerkstatt - Ausgangspunkt für

persönlichkeitsorientierte Professionalisierung in der Ausbildung

für Lehrpersonen

Verzeichnis der Autor*innen 


\title{
Sabine Fischer und Max de Baey-Ernsten
}

\section{Didaktische Perspektiven einer ästhetischen Werkstatt}

\begin{abstract}
Im Beitrag wird der Stand der Begleitforschung zur Entwicklung einer Werkstatt ästhetischer Erfahrung in einem kindheitspädagogischen Studiengang dargestellt. Die Werkstatt hat die Erprobung, Begleitung und Unterstützung subjektiver ästhetischer Erfahrungen von Studierenden zum Ziel. In einem doppeldidaktischen Sinn soll Studierenden die Möglichkeit geboten werden, Lernprozesse selbst zu erfahren und zu reflektieren.

Grundlegende theoretische Konzepte sind Deweys Überlegungen zu ästhetischer Erfahrung als das unmittelbar empfundene Verhältnis von Ordnung und Vollendung. In einem Verständnis ästhetischer Erfahrungen als basales Prinzip kindlichen Erlebens und Gestaltens von Welt besteht weiter ein Bezug zu Gerd Schäfer, der postuliert, dass ästhetische Tätigkeit bei Kindern beinhaltet, die Welt sinnlich zu erfassen, sie nachzuahmen, umzuformen und neuzugestalten.

Folgende Fragestellungen werden bearbeitet: Welche Dimensionen bilden sich in ästhetischen Erfahrungen von Studierenden ab? Wie können ästhetische Erfahrungen von Studierenden im Rahmen von Werkstattarbeit angeregt, begleitet, unterstützt und reflektiert werden? Wie können diese Erfahrungen zur Kompetenzentwicklung der Studierenden beitragen? In Kontexten mit Werkstattcharakter werden Studierenden Möglichkeiten ästhetischer Erfahrungen eröfnet. Ihre Perspektiven wurden durch Gruppendiskussionen, Fragebögen und Beobachtungsprotokolle erfasst. Mittels der Grounded Theory als handlungsleitende Forschungsstrategie wurden erste theoretische Fundierungen für zentrale Dimensionen einer ästhetischen Werkstatt vorgenommen. Diese Dimensionen werden dargestellt und diskutiert. Außerdem werden didaktische Perspektiven entwickelt, die darauf ausgerichtet sind, dass die Studierenden die Potenziale ästhetischer Erfahrungen für das Lernen der Kinder erkennen, für die aisthetischästhetischen Erfahrungen in der Perspektive der Kinder sensibilisiert werden und diese ggf. auch rekonstruieren können.
\end{abstract}




\section{Ausgangssituation}

Die Idee für eine ästhetische Werkstatt im Studiengang Bildung, Erziehung und Kindheit/ Childhood Studies der Evangelischen Hochschule in Darmstadt ist aus den folgenden Überlegungen entstanden:

Zum einen bringen viele Studierende des Studiengangs außer einem achtwöchigen Praktikum nur wenig Praxiserfahrung mit. Eine intensive Bearbeitung unterschiedlicher Inhalte bleibt dadurch stark theoriebasiert und der Transfer in die Praxis gelingt nur eingeschränkt. In der Werkstatt bieten sich Möglichkeiten zum Experimentieren, Sammeln von Erfahrungen und zur Reflexion, die dann in Bezug zu den Aufgaben und Anforderungen kindheitspädagogischer Professionalität gebracht werden können. Kaiser (vgl. 2016, S. 320) belegt in ihren Studien, dass durch die Werkstattarbeit Transferprozesse wie Selbst- und Peerreflexion, wiederholte Erfahrung, Wechselseitigkeit von Theorie und Praxis und der Umgang mit komplexen Situationen, in denen keinem Rezeptwissen gefolgt werden kann, möglich sind.

Ein weiteres Argument für die Werkstatt ist, dass frühkindliche Bildungsprozesse mit sinnlichen Wahrnehmungen beginnen. Folgt man den Erkenntnissen der Hirnforschung, tragen die daraus resultierenden Erfahrungen dazu bei, dass Kinder die Welt zunehmend differenzierter wahrnehmen können. Wahrnehmungsmuster entwickeln sich, die die Erfahrung der Welt, der Beziehung zu den Menschen, der Dinge und geistigen Phänomene sowie des eigenen Körpers strukturieren. Mit Hilfe der Sinne wird die Wirklichkeit so geordnet, dass man mit ihr umgehen, sich in ihr orientieren und schließlich über sie nachdenken kann (vgl. Schäfer, 2001, S. 86f). Ausgehend von der These, dass Ästhetik alle Ordnungen der Wirklichkeit umfasst, die mit Hilfe der Sinne geschaffen werden, bedeutet dies, dass ästhetische Erfahrungen in der Kindheit basal sind und in didaktische Überlegungen bei der Gestaltung von Bildungseinrichtungen und Prozessen umfassend einbezogen werden sollten. Ästhetische Erfahrungen sind demnach grundlegend und Voraussetzung für weitere Lern- und Bildungsprozesse. Sie müssen als Querschnittsthema in allen Bildungsbereichen Berücksichtigung finden und dürfen nicht isoliert betrachtet werden.

Zentral für die Gestaltung der Werkstatt und auch des Forschungsprojektes ist ebenso eine ethische Perspektive, die im Kontext der Childhood Studies und damit der Ausrichtung des Studiengangs entstanden ist. Diese ethische Orientierung versteht Kinder als Akteure mit ihrem Recht auf ein Leben in der Gegenwart. Damit zeigt sie auf, dass es nicht ausreicht, lediglich die Entwicklungs-, Lern- und Bildungsprozesse der Kinder auf die Zukunft gerichtet zu fokussieren, sondern es geht darum, die Kinder an allen sie betreffenden Belangen zu beteiligen und pädagogische Lebensräume gemeinsam mit ihnen in einer gegenwartsorientierten Perspektive zu gestalten. Ziel einer solchen Ethik ist, sowohl das Glück 
und das gute Leben der Kinder als auch das gerechte Zusammenleben in den Einrichtungen sicher zu stellen. Und diesem Anspruch soll auch die ästhetische Werkstatt entsprechen. Die Prozesse und Strukturen in der Werkstatt sind deshalb mit einem bestimmten Bildungsverständnis verknüpft. Dieses ist konstruktivistisch ausgerichtet und basiert auf intergenerationalen und dialogischen Prozessen, ist orientiert an Selbstbestimmung, Mitbestimmung und Solidaritätsfähigkeit und soll allen Kindern Bildungszugänge auf unterschiedlichen Ebenen ermöglichen. Die Kinder werden dabei in ihrer individuellen Existenzweise und in ihren Lebenswelten wahrgenommen.

Die ästhetische Praxis bietet die Möglichkeit, Welt- und Lebenserfahrungen zu artikulieren, neue Sichtweisen zu eröffnen und Lebens- und Handlungsmöglichkeiten sowie Handlungsspielräume zu erweitern. Kompetenzen wie Wahrnehmen und Erkennen werden verfeinert, Fantasie und Kreativität angeregt (vgl. Seel, 1991; S. 44ff.; Wolf, 1991, S. 111f.; Düwell, 1999, S. 281f. zit. nach Fenner 2013, o. S.). Sie trägt aber auch darüber hinaus zur Entlastung vom Alltagsstress bei, kann positive Gefühle des ästhetischen Wohlgefallens (vgl. Seel, 1996, S. 14f.) bieten und hat psychohygienische oder therapeutische Wirkungen, weil belastende Erfahrungen besser bearbeitet und leichter ertragen werden (vgl. Heimes, 2010, S. 40). Im Sinne eines gerechten Zusammenlebens eröffnet Ästhetik Kindern unterschiedliche Zugänge zu Bildung, unterschiedliche Möglichkeiten der Weltaneignung und verschiedeneAusdrucksmöglichkeiten. Ästhetisches Handeln und ästhetische Erfahrungen tragen dazu bei, sich in einer Welt zu verhalten und sich in ihr gestaltend zu bewegen und entsprechen damit einem inklusiven Bildungsverständnis, wie z. B. U. Stinkes (vgl. 2008, S. 100) es versteht.

\section{Zielsetzung der Werkstatt}

Der Schwerpunkt der Werkstatt liegt im künstlerisch gestaltenden und im wahrnehmenden Bereich. Handschick et al. (2018, S. 142) betonen, dass ästhetische Bildungsentwürfe in werkstattartigen Konzepten Lernformen anstreben, die ganzheitlich multisensorische Erfahrungen und damit körperlich-geistige Involviertheit ermöglichen. Dabei geht es um eine emotionale, reflexive und diskursive Auseinandersetzung sowie darum, bei den Studierenden eine bewusste, auch auf sich selbst bezogene Wahrnehmungshaltung zu unterstützen. In einem „doppeldidaktischen Sinn" (Kaiser, 2016, S. 275) soll Studierenden der Ev. HS Darmstadt die Möglichkeit gegeben werden, auf einem adäquaten Reflexionsniveau Lernprozesse selbst zu erfahren“ (ebd. S. 275).

Angestrebt wird ein subjektorientiertes Verständnis ästhetischer Prozesse und deren Wahrnehmung im Kontext von „Ich- und Welterfahrungen“ sowohl in ihrer Bedeutung für die Kinder als auch für die eigene Person. Außerdem geht 
es um ein tiefgehendes Verständnis für den hohen Grad der Identifikation mit den Prozessen und Produkten sowie den Herausforderungen und Krisen, die mit ästhetischem Handeln verbunden sind. Eingebunden in Prozesse des aktiven forschenden Lernens, des Wahrnehmens und Reflektierens soll die Praxis ästhetischen Handelns den Studierenden intensive aisthetische/ästhetische Erfahrungen eröffnen und sie für spezifische Thematiken im Kontext ästhetischer Bildung in der pädagogischen Praxis sensibilisieren. Die Anforderungen ästhetischer Praxis z. B. in Form von analytischen Fähigkeiten, Reflexions- und Entscheidungsfähigkeiten und weiteren für die Lösung ästhetischer Probleme relevanten Kompetenzen wie z. B. Handlungs- und Prozessorientierung sollen von den Studierenden erkannt und in ihrer Bedeutung für das pädagogische Handeln in der Praxis eingeschätzt werden. Die Werkstatt wird als Raum verstanden, der das Lernen der Studierenden herausfordert, begleitet, unterstützt und reflektiert und zur Professionalisierung der Studierenden im Bereich ästhetischer Bildung beiträgt.

Wie die Werkstatt als Ort gestaltet werden kann, an dem Kinder in ihrer Existenzweise als von Erwachsenen differenzielle Zeitgenossen (vgl. Hengst, 2013) anerkannt werden, stellt eine besondere Herausforderung dar. Das Wohlbefinden der Kinder im Blick zu haben und zu fragen, wie die Kinder in ihren je spezifischen und individuellen Zugangswegen und Bildungsprozessen unterstützt werden können, soll bei der Entwicklung der Werkstatt besonders fokussiert werden. Die Erarbeitung einer Didaktik ethisch-ästhetischer Dynamiken einer Bildung in der frühen Kindheit, die nicht nach Ergebnissen von Bildung fragt, sondern die ein Lernen mittels ästhetischer Prozesse anbahnen möchte, steht dabei im Mittelpunkt. Dieses Lernen impliziert die Neukonstruktion von Welt, vollzieht sich in individuellen Prozessen, erfolgt selbstreguliert, in sozialen und situativen Kontexten und entspricht einen kumulativen Prozess (vgl. Wedekind, 2009; VeLW, 2009; Kaiser, 2016).

Die Werkstatt soll sich dabei zu einem Ort gemeinsamer Interaktion und Wissenskonstruktion entwickeln, wobei der Exploration und Erforschung didaktischer Prinzipien in ästhetischen Lernkontexten besondere Bedeutung beigemessen wird.

\section{Grundlegende theoretische Konzepte}

Sowohl für die Gestaltung der Werkstattarbeit als auch für das Forschungsprojekt wurden zwei theoretische Konzepte ausgewählt, die handlungsleitend sein sollten, nämlich zum einen John Deweys pragmatische Lerntheorie, die oft als „learning by doing“ beschrieben wird, und sein Werk „Kunst als Erfahrung“; und zum anderen Gerd Schäfers theoretische Überlegungen zu Bildungsprozessen in der frühen Kindheit im Kontext der Reggio-Pädagogik. 
Deweys Arbeiten wurden gewählt, weil dieser sich in seinem Werk konsequent am Demokratiegedanken orientiert und damit den ethischen Anforderungen der Werkstatt entspricht, aber auch, weil sein Konzept des „learning by doing“ darauf basiert, dass Erkenntnis aus einem Prozess des Wahrnehmens eines Problems, der Beobachtung der gegebenen Tatsachen, der Formung und Ausarbeitung eines naheliegenden Schlusses und der handelnden Erprobung entsteht (Dewey, 2005, S. 203). Wissen und Erkenntnis werden somit in einem individuellen Prozess konstruiert, der für die Arbeit in einer Lernwerkstatt passend ist. Eine Erfahrung wird nach Dewey aber nicht nur von den Handlungen des Subjekts bestimmt, sondern auch von den durch Handlungen bestimmten Wahrnehmungen gesteuert. Deshalb sind es die Wahrnehmungen, die einer Erfahrung Inhalt verleihen (vgl. Dewey, 2018, S. 69). Somit misst Dewey den aisthetischen Prozessen und Erfahrungen, also den sinnlichen, körperlichen Wahrnehmungen und Empfindungen, in Bezug auf den Erkenntnisgewinn eine besondere Bedeutung bei und entspricht damit einem weiteren zentralen Anliegen der Werkstatt. Letztlich soll den Lernenden Deweys Konzept folgend

die Möglichkeit gegeben werden, durch eigene Erfahrungen und Handlungen sowie durch Partizipation und Teilhabe an gemeinsam mit anderen durchgeführten Aktivitäten und Projekten zu einer umfassend selbsttätigen und selbst bestimmten Entwicklung ihres Lernens zu gelangen. Demokratische Selbstbestimmungs-, Mitbestimmungs- und Solidaritätsfähigkeit beginnen für Dewey im Kleinen, sie sind eine Frage konkreter Handlungen, Teilnahmen und Beobachtungen von Lernenden im alltäglichen Miteinander. (Neubert, 2011, S. 221)

Dewey kommt nicht nur in ethischer Perspektive den Anforderungen an die Werkstatt nach, sondern auch in ganz besonderer Weise in einer konstruktivistischen, reflexiven und aktuellen elementardidaktischen Perspektive. Weitere Bezüge zu aktuellen kindheitspädagogischen Diskursen und zu Fragen der Lernwerkstattarbeit lassen sich auch in Deweys Vorstellungen zum ästhetischen Ausdrucksakt herstellen, den er als eine Verbindung von Merkmalen und Werten des aktuellen Daseins versteht, die mit vergangenen Erfahrungen in der Persönlichkeit aufgenommen werden (vgl. Dewey, 2018, S. 87). Wie auch in der kindheitspädagogischen Praxis und Lernwerkstattarbeit kommt in seinem Werk den Dimensionen Unmittelbarkeit und Individualität eine bedeutende Funktion zu, die auch für das Forschungsprojekt relevant sind, wenn es um die Frage geht, wie direkte, ursprüngliche Zugänge und biografische Erfahrungen der Akteure einbezogen werden können. Im gestalteten „Produkt“ bzw. Ausdrucksakt zeigt sich für Dewey die Neuschaffung des Erfahrungsmaterials, die „durch das unmittelbar empfundene Verhältnis von Ordnung und Vollendung gesteuert wird“ und dadurch den ästhetischen Charakter einer Erfahrung ausmacht. Eine ästhetische Erfahrung wird also dann erreicht, wenn eine gegenseitige Anpassung von Selbst und Objekt erkenn- 
bar wird (Dewey, 2018, S. 64). Dewey sieht im Ästhetischen deshalb eine Basiserfahrung für alle höheren Funktionen der Erfahrungen. Erkenntnisfähigkeit, Sinnlichkeit und Emotionalität sind für Dewey die grundlegenden Dimensionen der Erfahrung, wobei erst die Ästhetik Ordnung in die sinnlichen Erfahrungen bringt. Für Gerd Schäfer, der sich in seinen theoretischen Ausführungen auf Dewey bezieht, sind ästhetische Erfahrungen gleichfalls basal und grundlegend im Kontext frühkindlicher Bildung. Auch für ihn sind Handlungs- und Sinneserfahrungen der Ausgangspunkt von Erfahrungswissen.

Indem Kinder erfassen, wie die Dinge zusammenhängen, in welchen Kontexten sie sich im allgemeinen befinden, wie sie üblicherweise geformt sind und wozu sie gebraucht werden können, entsteht in ihren Köpfen einen sinnliche Ordnung der Wirklichkeit, in der sie bereits denken, bevor sie sprechen. Diese Erfahrungen durchlaufen Umwandlungen, bis sie schließlich symbolisch gefasst und sprachlich gedacht werden können. (Schäfer, 2009, S. 83f.)

Kinder ordnen und gestalten Wirklichkeit mit sinnlichen Mitteln. Für Schäfer ist dies eine Form des alltäglichen Denkens und der Ausgangspunkt für die „hundert Sprachen" der Kinder in der Reggio-Pädagogik, für hundert Möglichkeiten, die Welt zu verstehen, zu interpretieren, und die eigene Identität in den Beziehungen zu anderen auszudrücken. Die Reggio-Pädagogik geht davon aus, dass Kinder ihre Wirklichkeitserfahrungen be- und verarbeiten, indem sie gestalten und denken. Dafür stellt die Reggio-Pädagogik den Kindern gleich einer Lernwerkstatt einen Raum zur Verfügung - das Atelier. Dabei handelt es sich um einen Raum mit Material, welches das Kind anregen, herausfordern und unterstützen soll, selbsttätig und kompetent im Dialog mit anderen, seine „hundert Sprachen“ zu entwickeln (vgl. Stenger, 2010, S. 24 ff). Diese „hundert Sprachen“ der Kinder, das belegen Dokumentationen der Reggio-Pädagogik, sind zwar höchst individuell, doch auch kulturell geprägt, da es, wenn wir sprechen, auch darum geht, dass wir von anderen verstanden werden (vgl. Schäfer 2013, S. 43). Die Reggio-Pädagogik bildet somit eine geeignete Basis für die Entwicklung einer ästhetischen Werkstatt in einem kindheitspädagogischen Studiengang. Sie ist subjektorientiert und basiert darauf, dass Kinder in selbsttätigen Prozessen des Experimentierens und Gestaltens zu Erkenntnissen gelangen und ihre eigene Identität in Beziehung zur Welt entwickeln. Dabei konstruiert das Kind in einem bestimmten kulturellen Kontext seine eigene Wirklichkeit, die es in den „hundert Sprachen“ ausdrückt und weiter verarbeitet. Aufgrund dieser Fundierungen sichert die Reggio-Pädagogik für die Entwicklung der ästhetischen Werkstatt einen klaren Bezug zur Elementardidaktik und die Erkenntnisse, die aus dem Forschungsprojekt gewonnen werden, können mit bisherigen Überlegungen zur Qualifizierung von Studierenden in kindheitspädagogischen Studiengängen ebenso in Verbindung gebracht werden wie mit der Weiterentwicklung der Werkstattarbeit und der Elementardidaktik in der Kindheitspädagogik. 


\section{Forschungsdesign}

Besonders hervorzuheben ist der experimentelle Charakter des Projektes, denn die Werkstatt wird gemeinsam mit Studierenden, Kindern und Professionellen im Feld entwickelt. Dazu wurde in einem ersten Schritt im Studiengang ein Lehrforschungsprojekt „Ästhetische Werkstatt“ initiiert, an dem bisher über drei Semester hinweg eine Gruppe von sieben Studierenden teilgenommen hat. Mittels der Grounded Theory als Forschungsstrategie wurden erste durch das Lehrforschungsprojekt durchgeführte Aktivitäten sowie Lehrveranstaltungen im Bereich Gestaltung, die einen Werkstattcharakter hatten, in die Forschungsaktivitäten eingebunden. In dieser ersten Phase lag der Fokus auf der Werkstatt als Lernarrangement für die Studierenden. Die Ergebnisse, die in diesem Beitrag dargestellt werden, beziehen sich auf diese erste Projektphase, in der die Studierenden und ihre ästhetischen Erfahrungen im Vordergrund standen. Für die zweite Projektphase ist geplant, auch Kinder und pädagogische Fachkräfte einzubeziehen.

Zu Beginn des Lehrforschungsprojektes setzte sich die Forschungsgruppe mit den theoretischen Konzepten von Dewey und Schäfer sowie der Reggio-Pädagogik und weiterer Literatur zur ästhetischen Erfahrung auseinander, u. a. mit von Mattenklott und Mollenhauer, um zu einem Konsens darüber zu gelangen, was als ästhetische Erfahrung bezeichnet werden kann und welche Dimensionen in diesem Kontext einbezogen werden müssen. Aus dieser Diskussion ergaben sich folgende acht Thesen zu ästhetischer Erfahrung, die für das Forschungsprojekt handlungsleitend sind und auf die sich die Gruppe einigte:

1. Kinder sind ästhetisch tätig: Sie erfassen die Welt sinnlich und gestalten sie fantasievoll um und mit.

2. Aisthesis als sinnliche, körperliche Wahrnehmung und Empfindung ist grundlegend für ästhetische Erfahrung.

3. Ästhetische Erfahrungen sind subjektiv geprägt.

4. Ästhetische Objekte stellen eine Verbindung zwischen der Innenwelt des Wahrnehmenden und dem gestalteten Phänomen der Außenwelt dar.

5. Ästhetische Erfahrungen sind im Alltag, aber auch als Ereignis existent.

6. Eine Kultur aisthetischen/ästhetischen Lernens erfüllt eine Vielzahl pädagogischer Aufgaben.

7. Ästhetische und aisthetische Prozesse bedingen Reflexionsfähigkeit

8. Der Terminus ästhetische Bildung widerspricht dem Charakter des Ästhetischen.

Letztere doch provokante These bedarf möglicherweise der Erläuterung: In der Auseinandersetzung mit den Themen ästhetische Erfahrung und Bildung kamen die Studierenden in einer kritischen Perspektive zu dem Schluss, dass Bildung in vielen Konzepten und u. a. auch in den Bildungsplänen der Länder in der Regel darauf zielt, dass die Kinder in den Einrichtungen Kompetenzen erwerben, die 
den curricularen Erfordernissen der Schule entsprechen oder gar auf ein höheres Ziel hin, nämlich die potentielle Mündigkeit, ausgerichtet sind. In beiden Fällen werden Kinder nicht in der Gegenwart wahrgenommen, sondern in die Zukunft gedacht. Mit diesem häufig vorgefunden Bildungsverständnis wird negiert, dass Kinder ihre Bildungsprozesse eigenständig vorantreiben, indem sie i. S. eines „learning by doing“" an für sie bedeutungsvollen und mit Sinn aufgeladenen Themen arbeiten; noch wird bedacht, dass Mündigkeit als Entwicklungsziel von manchen Kindern aufgrund ihrer spezifischen Entwicklungsbedingungen gar nicht erreicht werden kann. Ästhetische Prozesse wie sie von Dewey oder Schäfer und der Reggio-Pädagogik beschrieben werden, sind mit diesen Vorstellungen jedoch nur schwer vereinbar und widersprechen diesen sogar.

Das Forschungsprojekt soll darüber Aufschluss geben, wie eine Lernumgebung im Format einer Werkstatt gestaltet werden kann, die die Prozesse ästhetischer Erfahrung von Kindern unterstützt. Dafür wurde folgende Forschungsfrage entwickelt:

Wie sollte eine Werkstatt ästhetischer Erfahrungen in einem kindheitspädagogischen Studiengang gestaltet sein, die das Lernen der Studierenden und der Kinder herausfordert, begleitet, unterstützt und reflektiert und zur Professionalisierung der Studierenden im Bereich ästhetischer Bildung beiträgt?

Da der Fokus in dieser ersten Phase auf die Werkstatt als Lernarrangement für die Studierenden gerichtet war, hat sich die Forschungsgruppe besonderes mit der Frage beschäftigt, welche Erfahrungen die Studierenden im Rahmen dieser Arrangements machen und unter welchen Bedingungen es möglich ist, dass die Studierenden ihre Erfahrungen zur Weiterentwicklung ihrer Professionalität im Bereich ästhetischer Bildung nutzen können. Mit dem Ziel, daraus erste didaktische Perspektiven zu entwickeln, wurden von den Studierenden im Projekt in Bezug auf die Forschungsfrage weitere Differenzierungen vorgenommen, die sich in folgenden Fragestellungen widerspiegeln:

- Welche Dimensionen bilden sich in ästhetischen Erfahrungen von Studierenden ab?

- Wie können ästhetische Erfahrungen von Studierenden im Rahmen von Werkstattarbeit angeregt, begleitet, unterstützt und reflektiert werden?

- Wie können diese Erfahrungen zur Kompetenzentwicklung der Studierenden beitragen?

o Wie gelingt eine Sensibilisierung der Studierenden für die aisthetisch-ästhetischen Erfahrungen in der Perspektive der Kinder?

○ Welche Potenziale ästhetischer Erfahrungen für das Lernen der Kinder können die Studierenden in der Reflexion ihrer eigenen Werke rekonstruieren? 


\section{Datenerhebung}

Die Grounded Theory wurde als Forschungsstrategie ausgewählt, um erste theoretische Perspektiven für die Didaktik der Werkstatt zu entwickeln. Im Sinne des Theoretical Sampling stand dabei die Auswahl der Stichproben zu Beginn noch nicht fest, sondern entwickelte sich im Forschungsverlauf parallel zur Analyse und den Auswertungsschritten (vgl. Strübing, 2019, S. 531). Die erste Datenerhebung erfolgte im Anschluss an eine Lehrveranstaltung zum Thema "Gestaltung", die zum Ziel hatte, dass Studierende aisthetische Wahrnehmungs- und ästhetische Erfahrungsformen als Bezugspunkte für Lernen und Bildung zunächst im eigenen Erleben erkennen und vor dem Hintergrund von Theorien und Methoden, die in anderen Lehrveranstaltungen des Moduls erarbeitet wurden, zu reflektieren. In der Abschlussreflexion der Veranstaltung sollten die Studierenden vier Reflexionsfragen bearbeiten und dann ihre Ergebnisse visualisiert im Plenum vorstellen. Daran anschließend galt das Erkenntnisinteresse den ästhetischen Erfahrungen der Studierenden des 1. Semesters. In einer Einführungsveranstaltung ins Studium setzten diese sich in Gruppen mit mehr oder weniger strukturierten Gestaltungsaufgaben auseinander. Gruppe A sollte eine Skulptur kreieren, die rollt, und Gruppe B eine Murmelbahn, die möglichst kreativ gestaltet ist. Den Studierenden standen hierfür verschiedene bedeutungsoffene Materialien zur Verfügung. Im Anschluss daran wurden sie mittels eines Fragebogens zur Reflexion über den Prozess angeregt. Begleitend wurden Beobachtungen durchgeführt und protokolliert. Da dieses Angebot stark von kooperativen und gruppendynamischen Einflüssen bestimmt war, sollten daran anschließend kontrastierend Selbsterfahrungen von Studierenden in den Forschungsprozess eingebunden werden. Ein Anliegen war dabei, die ästhetische Erfahrung als Mikroprozess besser zu verstehen. Hierzu wurden Einzelarbeiten mit methodischer Vorgabe (Arbeit mit Fingerfarben) durchgeführt. Es wurde ein Fotoprotokoll und im Anschluss daran eine schriftliche narrative Selbstreflexion der Studierenden erstellt.

\section{Darstellung der Ergebnisse}

Bevor im Folgenden aus diesen Daten erste theoretische Fundierungen für zentrale Dimensionen einer ästhetischen Werkstatt dargestellt werden, erscheint es sinnvoll, kurz auf den Auswertungsprozess einzugehen.

Die erste Phase der Auswertung der Daten, das „Offene Kodieren“ (Strübing, 2019, S. 535), fand in der Gruppe des Lehrforschungsprojektes statt. Hierbei wurde zunächst eine induktive Vorgehensweise gewählt, um allen Aspekten offen gegenüberzustehen. Im Anschluss daran wurden mögliche Verknüpfungen von 
Codes hergestellt. In einem weiteren gemeinsamen Prozess wurde das Codesystem nochmals überarbeitet und dabei wurden zentrale Kategorien entwickelt, die zur Klärung der Fragestellungen geeignet schienen. Dabei entstanden die Kategorien „subjektive Erfahrung“, „Rahmenbedingungen“ und „Didaktik“. Nach dem offenen Kodieren folgte das axiale Kodieren, hier wurden das untersuchte Material und die ersten Ergebnisse nach „den Bedingungen, den Interaktionen zwischen den Akteuren, den Strategien und Taktiken [und] den Konsequenzen“" (Strübing, 2019, S. 537) kodiert. Im Abschließenden letzten Schritt, dem selektiven Kodieren, ging es darum, einen roten Faden zu identifizieren, um die Fragestellung zu beantworten. Hierzu wurde anhand der Kernkategorie „Wie kann eine ästhetische Werkstatt in einem kindheitspädagogischen Studiengang dazu beitragen, Studierende zu befähigen, ästhetische Erfahrungen von Kindern wahrzunehmen, zu begleiten und zu unterstützen“ das gesamte Material rekodiert und „auf eine einheitliche Analyseperspektive hin überarbeitet" (ebd., S. 538). Dabei wurden folgende Dimensionen entwickelt, die für die Kernkategorie zentral sind, hier aber nur in stark komprimierter Form dargestellt werden können.

\subsection{Dimension ästhetischer Erfahrungen}

In der Dimension ästhetische Erfahrungen werden Aussagen von Studierenden zu ihren ästhetischen Erfahrungen und den dazugehörigen Empfindungen analysiert. Dabei konnten folgende Subkategorien, die für die ästhetischen Erfahrungen der Studierenden relevant sind, gebildet werden: Emotion, Exploration, Subjektivität, Aisthesis und Ergebnis.

Es zeigt sich in den Aussagen, dass das emotionale Befinden der Schaffenden für den ästhetischen Prozess und die ästhetischen Erfahrungen von Bedeutung ist. Beispielsweise scheint es sehr wichtig zu sein, dass die Studierenden ein Gefühl der Autonomie erleben und positive Gefühle wie Spaß und Freude bei sich und anderen sehen.

Also wir haben viele kreative Prozesse erlebt, dadurch, dass wir viel Freiraum hatten und wir ausprobieren durften, ähm, wir hatten dann ausreichend Zeit, es hat uns auch Spaß gemacht, wie gesagt, weil wir uns frei entfalten konnten. (Gruppenreflexion Seminar Gestaltung, n4)

Vor allem das Ausprobieren und die Exploration scheinen für die ästhetische Erfahrung von besonderer Bedeutung. Manche Studierende berichteten in diesem Zusammenhang z. B. davon, wie hilfreich es war, wenn es ihnen gelang, ohne einen bestimmten Plan zu verfolgen in die Arbeit einzusteigen und den Kopf auszuschalten: 
Ich habe anfangs erwartet, dass wir zunächst einmal zögerlich an die Sache rangehen und uns erst einmal überlegen, wie die Skulptur aussehen soll. Dabei haben wir, wider Erwarten, einfach angefangen zu „tun“. (Fragebogen Einführungsveranstaltung, n9)

Auch der Einfluss von Vorerfahrungen und Gewohnheiten spielt im Kontext ästhetischer Erfahrungen eine bedeutende Rolle, denn fast alle Studierenden haben bereits Erfahrungen mit bestimmten Materialen und Wahrnehmungen (visuelle, haptische, auditive) sowie Gestaltungsprozessen, die mit Emotionen verknüpft sind. Dabei zeigt sich beispielsweise, dass ein negatives Selbstbild in Bezug auf das eigene kreative Potenzial einem freien entspannten und unvoreingenommenen Agieren entgegenstehen kann. Wie das folgende Zitat belegt, fiel es den Studierenden schwer, nicht der üblichen Gewohnheit zu folgen und sofort sowohl das eigene als auch die Werke der anderen zu bewerten. Dennoch konnten sie nachvollziehen, welche Bedeutung dieses Vorgehen für die eigene ästhetische Erfahrung hat.

Das, was auch gut gewesen ist, dass man halt nicht denken musste, wo man gearbeitet hat, weil das erste Mal, wo wir mit Farbe gearbeitet haben, wir haben ja irgendwas gemacht und es sollte auch nichts, also wir sollten es auch nicht mit unseren Normen und Werten verbinden und keiner sollte auch sagen, ob das jetzt einem gefällt oder nicht gefällt, es ging einfach nur darum, dass wir uns frei entfalten konnten. (Gruppenreflexion Seminar Gestaltung, n3)

Den Einfluss normativer Vorstellungen auf die aisthetische Wahrnehmung und nachfolgend auf Gestaltungsobjekte bzw. das Gestaltungsergebnis zeigt auch folgendes Zitat:

Im ersten Moment habe ich mich zunächst auf das Gefühl der „schmierigen“ Farbe konzentriert, dann habe ich eher darauf geachtet, was auf meinem Bild war, hier habe ich dann auch sehr danach geschaut, was die anderen so malen und fand, mein Bild war lediglich ein schmiergier Klecks. (Narrative Selbstreflexion, n1)

\subsection{Dimension Kontext}

In der Dimension Kontext wurden jene Codes erfasst, die Aussagen der Studierenden zur Umgebung und zu den Kontextbedingungen der ästhetischen Prozesse beinhalten. Gruppendynamik, Zeit, Bewertung und Leistungsdruck sowie Atmosphäre konnten hier als Subkategorien analysiert werden.

Ästhetische Erfahrungen können durch Kontextbedingungen anregt, aber auch eingeschränkt werden. Im oben aufgeführten Zitat zeigt sich z. B., dass Vergleiche mit anderen bedeutsam sind. Die Gruppe als Kontext ästhetischer Erfahrungen kann ein großes Potenzial bereitstellen, doch nur dann, wenn es bei Gruppenarbeiten gelingt, das kreative Potenzial der einzelnen Mitglieder zu integrieren bzw. 
den Einzelnen herauszufordern, seine Ideen für ein großes gemeinsames Ganzes bereitzustellen.

Die Kooperation war sehr gut, da wir auf die Ideen der einzelnen eingegangen sind und Ideen weiterentwickelt haben. (Fragebogen Einführungsveranstaltung, n2)

Auch die Beteiligungsmöglichkeiten sowie die Rahmenbedingungen, wie z. B. die Gruppengröße, sind dabei von Belang. So kann eine zu große Gruppe zum Beispiel für einzelne überfordernd wirken und dazu führen, dass sie sich eher passiv verhalten:

Für mich waren es zu viele Gruppenmitglieder, wodurch der Prozess eher an mir vorbeizog. (Fragebogen Einführungsveranstaltung, n17)

Der Herausforderung von zu großen Gruppen begegneten die Teilnehmenden teilweise mit der Herausbildung von Kleingruppen. Die Kooperation in diesen wird als positiv wahrgenommen und unterstützt die kreativen Prozesse. Voraussetzung hierfür ist jedoch eine intensive Kommunikation und Absprache. Ist die Atmosphäre in der Gruppe auf eine kreative Lösung der Aufgabe ausgerichtet, werden Ideen ausprobiert, sind fokussierte Einzelarbeiten möglich. In einer solchen Atmosphäre, in der ein reger Austausch stattfindet und gemeinsamen gelacht wird, lassen sich Prozesse des Ausprobierens und Scheiterns beobachten:

Insgesamt beobachte ich einen Prozess, der sich maßgeblich über Ausprobieren und wieder Verwerfen von Ideen und Varianten gestaltet. Die meisten Studierenden sind ganz im „Tätigsein“. Drei Frauen arbeiten still - sie gestalten kleine Plastikröhrchen, die sie mit Knete-Schlangen umwickeln und dann auf den Tisch legen, ganz in die Nähe der ideengebenden Studentin. Ein Mann umwickelt ein größeres Styropor-Ei komplett mit Knete. Die Assoziation „Dino-Ei“ wird geäußert und auch beibehalten. Das DinoEi wird als Nase angebracht, fällt aber sofort wieder ab und wird erst mal beiseitegelegt. Ebenso die Kronkorken-Augen und der Mund. (Beobachtungsprotokoll Einführungsveranstaltung Gruppe A, Abs. 10)

Zur Atmosphäre tragen jedoch nicht nur Gruppensituation und Gruppendynamik bei, sondern auch die räumliche Atmosphäre eines offenen Ateliers wirkt inspirierend. Der Raum und der „Appell der Dinge“ (Stieve, 2008) fordern zu Handlungen heraus, wecken Gefühle, ziehen uns an, können uns aber auch abschrecken (ebd., S. 12).

Ja die Atmosphäre war natürlich in diesem Atelier ganz eine andere als in so einem normalen Lehrraum, also weil man da einfach, diese ganzen Kunstgegenstände und Materialien direkt in Verbindung sieht und diese Atmosphäre auch in diesen Arbeitstechniken eine ganz andere ist als in einem normalen Raum. (Gruppenreflexion Seminar Gestaltung, n5) 


\subsection{Dimension Didaktik}

Die Dimension Kontext zeigt z. T. Überschneidungen mit der Dimension Didaktik, da beide den Einfluss von Rahmenbedingungen außerhalb des Subjektes beschreiben. In dieser Dimension wurden aus den Codes die Subkategorien Vorgaben, Techniken, Rolle der Lehrenden und Anregung/ Inspiration gebildet, um verschiedene didaktische Einflüsse differenziert zu betrachten.

Die bisherigen ästhetischen Erfahrungen der Studierenden sind vielfältig, individuell und subjektiv. In einer Werkstatt für ästhetische Erfahrungen kann durch eine methodisch-didaktische Gestaltung der (Lern-)Situation und der Kontextbedingungen an den subjektiven Vorerfahrungen (z. B. Kindheitserinnerungen) der Studierenden angeknüpft werden.

In welcher Weise Dozierende Anregungen einbringen, Impulse setzen, Methoden und Beispiele vorstellen, als Lernbegleitende in Krisen unterstützen oder den Studierenden individuelle Freiräume eröffnen, hat dabei Einfluss auf den ästhetischen Erfahrungsprozess. Wie folgendes Zitat zeigt, ermöglicht Zurückhaltung mit Kritik, Korrektur und Leistungsdruck eine individuelle Entwicklung der ästhetischen Erfahrung für die Studierenden.

Frau $\mathrm{X}$ war eben auch immer wieder da, um zu gucken, falls irgendjemand irgendwo hängt oder nicht weiterkommt, fanden wir gut, dass sie ihre Vorschläge jetzt nicht aufgedrängt hat, aber immer wieder in den Raum kam und gefragt hat, gibt's irgendjemanden, der Input möchte oder der, äh, eine neue Perspektive braucht. (Gruppenreflexion Seminar Gestaltung, n2)

Für die Studierenden war es weiter hilfreich, wenn durch die Dozierenden ein erster Überblick über die Möglichkeiten bezüglich Materialien und Techniken erfolgte, die sie dann mit Videos, Fachbüchern, Bildbände etc. weiter vertiefen konnten.

[...] dass der Input [...] viel gebracht hat, also als Frau X [Dozentin] die ganzen Methoden vorgestellt hat, weil eben nicht jeder mit Aquarell oder Acryl gearbeitet hat, war's hilfreich, dass vorher mal alles kurz erklärt wurde und Beispiele gezeigt wurden. Das man auch sieht, wie kann das Ganze am Ende aussehen, ähm, das hat uns eher ein bisschen inspiriert, dass wir auch dann sofort entscheiden konnten, was wir als erstes machen wollen. (Gruppenreflexion Seminar Gestaltung, n2)

Didaktisch, das zeigen die bisherigen Ergebnisse, ist es von Vorteil, wenn sowohl individuelle Einzelarbeiten als auch Angebote in Gruppen-Settings möglich sind. Dadurch kann der Subjektivität ästhetischer Erfahrungen entsprochen werden. Denn kooperative Gruppensituationen können herausfordernd sein und ästhetische Erfahrungen verhindern, aber auch inspirieren und dynamisch, kreativ, und explorativ erlebt werden. Ebenso bergen das Setzen eines bestimmten Themas (Aufgabe) aber auch experimentelle Freiräume wie z. B. die autonome Wahl von 
Material und Technik für die einzelnen Personen individuelle Chancen und/oder Herausforderungen.

Wir fanden es ziemlich cool, dass es so viele offene, öffentliche Möglichkeiten, [stöhn], Haufen Möglichkeiten gab, also, dass man so vielfältig da sein konnte. [...] Weil bei mir war das so, ich hab jetzt bei diesem Drucken am Ende das mit Linoleum versucht, das hab ich dann irgendwie durch die ganze Zeit fertig gemacht und hab mich am Ende dann auch ein bisschen verzettelt und konnte dann nichts Neues mehr machen und ja, deswegen haben wir gesagt, manchmal war es auch echt anstrengend. (Gruppenreflexion Seminar Gestaltung, n8)

\subsection{Dimension Kompetenz/Professionalität}

Bei der Dimension Kompetenz/Professionalität stand die Frage im Mittelpunkt, welche Kompetenzen die Studierenden durch die Angebote mit Werkstattcharakter entwickeln bzw. weiterentwickeln können. Aus dem vorliegenden Material wurden für diese Dimension die Subkategorien Perspektivübernahme, Bild vom Kind(lichen Lernen) und Bild vom Kind als pädagogisches Objekt gebildet.

Das Kennenlernen und eigenständige „Beforschen“ ästhetischer Praxis sowie das Reflektieren und Explizieren eigener ästhetischer Erfahrungen soll Studierende dabei unterstützen, Kompetenzen im Bereich der ästhetischen Bildung zu entfalten. Dazu sollten sie die Prozesse, in denen Kinder ästhetische Erfahrungen machen, erkennen, antizipieren und ggf. verstehen können. Sie sollten in der Lage sein, anregende Situationen und Räume gemeinsam mit den Kindern zu gestalten, die ihren Bedürfnissen nach aisthetischer/ästhetischer Erfahrung entsprechen. Die Perspektive der Kinder zu übernehmen und das eigene Bild vom Kind zu reflektieren bzw. weiterzuentwickeln, sind Kompetenzen, die dabei eine zentrale Rolle spielen.

Die Studierenden beschreiben, dass sie durch die eigene tätige Auseinandersetzung bei Gestaltungsprojekten die Perspektiven der Kinder besser nachvollziehen und sich besser in die Rolle der Kinder hineinversetzen können, wie in folgenden Antworten einer Nachbefragung von Studierenden zum Zusammenhang zwischen eigenen Erfahrungen mit einem Gestaltungsangebot und pädagogischer Professionalität deutlich wird:

Sich einmal in die Rolle/Perspektive des Kindes zu versetzen hilft einem, selbst eine andere Denkweise einzunehmen und die der Kinder eventuell auch besser zu verstehen. (Fragebogen Einführungsveranstaltung, n32)

Sich in ein Kind hineinzuversetzen. $\mathrm{Zu}$ schauen wie man gewisse Situationen selbst behandelt und dann vergleichen wie ein Kind dies macht. (ebd., n29)

Ich denke, dass Kinder viel mehr durch Ausprobieren erforschen und einem solche Aufgaben dabei helfen, das besser nachvollziehen zu können. (ebd., n28) 
In den Aussagen lassen sich auch Hinweise auf das Bild der Studierenden vom kindlichen Lernen ableiten, wobei hier besonders der explorative Charakter des kindlichen „Tätigseins“ betont wird. Dieses beschreiben sie mit folgenden Eigenschaften:

Winfach drauf los bau[en] und sich nicht stundenlang den Kopf zerbrechen (ebd., n27)

Sich auf den kreativen Fluss einlassen (ebd., n14)

Sie [Kinder] probieren Dinge erst einmal und gucken ob, oder wie es funktioniert. (ebd., n19)

Viel mehr durch Ausprobieren erforschen (ebd., n28)

Andere Studierende hingegen leiten aus ihren Erfahrungen ab, dass Kinder in der tätigen Auseinandersetzung z. B. Fähigkeiten in folgenden Bereichen entwickeln: „Achtsamkeit erlernen, Teamfähigkeit, Geduld, logisches und räumliches Denken, lernen Angefangenes zu beenden und Konzentration" (ebd., n42). Besonders hervorgehoben wird auch die Bedeutung des Handelns für die Kreativität (ebd., n1, n2, n21\&22, n23, n44) sowie die Selbsterfahrung und das Selbstlernen.

In den Aussagen der Studierenden zeigt sich somit ein Spannungsverhältnis zwischen dem Nacherleben der Erfahrungen der Kinder und einem erwachsenenzentrierten Bildungsanspruch, der sich in den Aufzählungen von Lerninhalten und Möglichkeiten widerspiegelt. Ob der Zugang zur Perspektive der Kinder durch Reflexion explorativer Gestaltungsprozesse und der damit verknüpften leibsinnlichen aisthetischen Wahrnehmungen oder auch durch das Anknüpfen an biographische Kindheitserfahrungen sowie theoretische Modelle für alle Studierenden möglich wird, müssen weitere Forschungsaktivitäten zeigen.

\section{Entwicklung didaktischer Perspektiven}

Um die einzelnen Dimensionen in ein umfassendes theoretisches Konzept zu integrieren, wurde eine Kernkategorie gebildet, in der sich die Zielsetzung der Werkstatt wie folgt widerspiegelt. Eine ästhetische Werkstatt in einem kindheitspädagogischen Studiengang kann dazu beitragen, Studierende zu befähigen, ästhetische Erfahrungen von Kindern wahrzunehmen, zu begleiten und zu unterstützen.

Die ersten Ergebnisse der Studie zeigen, dass in einer ästhetischen Werkstatt Studierende ästhetische Erfahrungen sammeln können, wobei die ästhetischen Vorerfahrungen der Studierenden einbezogen werden müssen. Emotion und Exploration spielen für die Prozesse und daraus resultierende Erfahrungen eine zentrale Rolle. Durch den Kontext (Atmosphäre, Gruppendynamik, Zeit und Raum) sowie die weitere didaktische Gestaltung der (Lern-)Situationen wie z. B. 
das Handeln der Dozierenden werden die ästhetischen Erfahrungen der Studierenden angeregt und inspiriert oder können im negativen Fall auch behindert und eingeschränkt werden.

Die Reflexion dieser Prozesse und (Selbst-)Erfahrungen in der Werkstatt führen in einem doppeldidaktischen Sinn dazu, dass die Studierenden die Prozesse und Erfahrungen der Kinder beim Gestalten wahrzunehmen lernen. Dies lässt sich beispielsweise durch das Aufzeigen der Parallelen zwischen ihren Vorstellungen vom kindlichen explorativen Tätigsein und ihren eigenen explorativen Erfahrungen herstellen. Dadurch sind die Studierenden zunehmend in der Lage, die subjektive Perspektive der einzelnen Kinder sensibler nachzuvollziehen. Sie erkennen Kinder als Akteure, die sich in ästhetischen Prozessen die Welt aneignen und sie mitgestalten. Ihr Bild vom Kind im Kontext der neueren Kindheitspädagogik kann sich ausdifferenzieren und sie entwickeln erste didaktische Perspektiven, die sie aktuell und zukünftig mit entsprechender Unterstützung durch Lehrende mit theoretischem Wissen verknüpfen und mittels theoretischer Modelle weiter ausdifferenzieren können. Dadurch trägt die Werkstatt in einer besonderen Weise dazu bei, die Kompetenz und die Professionalität der Studierenden weiter zu entwickeln.

\section{Fazit}

Ästhetische Praxis ermöglichte den Studierenden ein „learning by doing“. Im Ausdrucksakt greifen sie auf Vorerfahrungen wie biografische Kindheitserinnerungen zurück und verbinden Vergangenes mit Gegenwärtigen. Dies basiert auf einer auf sich selbst bezogenen Wahrnehmungshaltung, die mit ästhetischen Erfahrungen einhergeht. Im Sinne kindheitspädagogischer Professionalität soll in Interaktionen mit Kindern eine neugierige Forschungshaltung im Sinne des Nicht-Wissens eingenommen werden. Dafür, und auch um weiteren Zielen der Werkstatt zu entsprechen, ist es wichtig, den Studierenden Methoden an die Hand zu geben, ihre ästhetischen Erfahrungen zu explizieren. Selbstwahrnehmungsprozesse in Form von Emotionen, Assoziationen und Gedanken während des ästhetischen Prozesses können beschrieben, mit Audiogeräten aufgezeichnet oder im Partner-Interview dargestellt werden. Dieses Vorgehen i. S. einer forschenden Haltung gegenüber den eigenen Wahrnehmungen und der Anerkennung dieser durch die eigene Person und/oder andere kann dazu beitragen, dass die Studierenden diese Erfahrungen und Erkenntnisse in den Kontext der pädagogischen Praxis übertragen.

Ästhetische Erfahrungen können komplexe neuartige Erfahrungen darstellen, in denen Prozess- und Handlungsorientierung im Vordergrund stehen. Durch Vergleiche zwischen den eigenen Prozessen und jenen der Kinder wird es möglich, 
dass die Studierenden die Bedeutung der Gegenwart sowohl für das eigene Handeln als auch das Handeln der Kinder erkennen. Diese prozess- und handlungsorientierte Haltung unterstützt die Entwicklung ethischer Orientierungen, die die Selbsttätigkeit der Kinder im Hier und Jetzt in den Vordergrund rücken. Auch die hohe Bedeutung des kindlichen Wohlbefindens und der Beteiligung von Kindern in allen Lebenslagen kann durch die Reflexion der Gelingensbedingungen eigener ästhetischer Erfahrungen ermöglicht werden. In zukünftigen Erhebungen sollte jedoch noch umfassender geklärt werden, welche Formen der Reflexion unter welchen Bedingungen dazu beitragen, den Zielen der Werkstatt zu entsprechen. In den Erhebungen insbesondere zu den Gruppenarbeiten zeigte sich, dass Partizipation und Teilhabe für die Studierenden eine wichtige Rolle spielen und die Studierenden Deweys Idee entsprechend ihr Lernen selbsttätig und in Selbstbestimmung weiter vorantreiben können. Werden die didaktischen Überlegungen außerdem darauf ausgerichtet, Individualität und Subjektivität ästhetischer Erfahrungen in den Mittelpunkt zu stellen, wird es möglich, in der Werkstatt ein inklusives Bildungsverständnis zu etablieren, das sowohl Kindern als auch Erwachsenen individuelle Zugangswege zu ihren Erfahrungen erlaubt und Verknüpfungen zur Idee der „hundert Sprachen“ des Kindes eröffnet.

Für den weiteren Projektverlauf, wird es jedoch zukünftig darum gehen, auch die Kinder in die Werkstattarbeit einzubeziehen. Dann sollten insbesondere Kooperation und Dialog zwischen Erwachsenen und Kindern im Rahmen von Werkstattarbeit in den Blick genommen werden. Auch der Reflexion der Potenziale einer Werkstatt in der Perspektive von Kindern und Erwachsenen und der Frage, wie ästhetische Erfahrungen von Studierenden dazu beitragen, die Perspektive der Kinder in ästhetischen Prozessen zu reflektieren, soll weiter nachgegangen werden. Perspektivisch sollte ein umfassendes didaktisches Konzept für die Werkstattarbeit in kindheitspädagogischen Studiengängen entwickelt werden und die ästhetische Werkstatt sollte modulübergreifend in den Studiengang implementiert werden, wobei sich inhaltlich Brücken zu Inhalten anderer Module schlagen lassen, z. B. zu Entwicklungspsychologie, Pädagogik, Diversität oder Kinderrechten.

\section{Literatur}

Dewey, J. (2005). Erfahrung und Natur. Suhrkamp.

Dewey, J. (2018). Kunst als Erfahrung (9. Aufl.). Suhrkamp. (Erstveröffentlichung 1934)

Düwell, M. (1999). Ästhetische Erfahrung und Moral. Zur Bedeutung des Ästhetischen für die Handlungsspielräume des Menschen. Alber.

Fenner, D. (2013/2012). Ethik und Ästhetik. Kulturelle Bildung Online. https://www.kubi-online.de/ artikel/ethik-aesthetik.

Handschick, M., Biard, E., Delitta-Möller, L. \& Möller, A. (2018). Ästhetische Bildung im Spiegel von Lernwerkstattkonzepten: Überlegungen zu interdisziplinären und übertragbaren Formaten der Kulturvermittlung für heterogene Lerngruppen. In M. Peschel \& M. Kelkel (Hg.), Fachlichkeit in Lernwerkstätten (138-152). Klinkhardt. 
Heimes, S. (2010). Künstlerische Therapien: Ein intermedialer Ansatz. Vandenhoeck \& Ruprecht.

Hengst, H. (2013). Kindheit im 21. Jahrhundert: Differenzielle Zeitgenossenschaft. Beltz Juventa.

Kaiser, L. S. (2016). Lernwerkstattarbeit in kindheitspädagogischen Studiengängen. kopaed.

Neubert, S. (2011). John Dewey (1859-1952): Erziehung zur Demokratie. In Dollinger (Hg.), Klassiker der Pädagogik (221-246). VS Verlag.

Schäfer, G. (2009). Frühe Wege ins Naturwissen. In G. Schäfer, H. Alemzadeh, Eden H. \& D. Rosenfelder (Hg.), Natur als Werkstatt (81-95). Verlag das Netz.

Schäfer, G. E. \& Beek, A. von der (2013). Didaktik in der frühen Kindheit: Von Reggio lernen und weiterdenken. Verlag das Netz.

Schäfer, G. E. (2001). Prozesse frühkindlicher Bildung. Vorlesung. typoskript. https://www.hf.unikoeln.de/data/eso/File/Schaefer/Prozesse_Fruehkindlicher_Bildung.pdf

Seel, M. (1996). Ethisch-ästhetische Studien. Suhrkamp.

Stenger, U. (2010). Zur Didaktik der Reggiopädagogik. In D. Kasüschke (Hg.), Didaktik in der Pädagogik der frühen Kindheit (114-144). Carl Link.

Stinkes, U. (2008): Bildung als Antwort auf die Not und Nötigung, sein Leben zu führen. In Fornfeld, B. (Hg.) Meneschen mit komplexer Behinderung (82-107). Reinhardt.

Strübing, J. (2019). Grounded Theory und Theoretical Sampling. In N. Baur \& Blasius J. (Hg.), Handbuch Methoden der empirischen Sozialforschung (2. Aufl., 525-544). Springer Fachmedien.

Verbund europäischer Lernwerkstätten e. V. (2009). Positionspapier des Verbundes europäischer Lernwerkstätten (VeLW) e.V.zu Qualitätsmerkmalen von Lernwerkstätten und Lernwerkstattarbeit. https:// www.forschendes-lernen.net/files/eightytwenty/materialien/VeLW-Broschuere.pdf

Wedekind, H. (2009). Lehre und Studium als eigenes Praxisfeld begreifen. Die Grundschulwerkstatt der Humboldt-Universität zu Berlin. Kontexis 30/2009, 4-5.

Wolf, U. (1991). Kunst, Philosophie und die Frage nach dem guten Leben. In Koppe, F. (Hg.): Perspektiven der Kunstphilosophie (109-132). Suhrkamp. 